Revue française de la traduction

\title{
La traduction de qualité : un point de vue du Bureau Permanent de la Conférence de La Haye de droit international privé
}

\section{Céline Château}

\section{OpenEdition}

\section{Journals}

Édition électronique

URL : http://journals.openedition.org/traduire/1295

DOI : $10.4000 /$ traduire. 1295

ISSN : 2272-9992

Éditeur

Société française des traducteurs

\section{Édition imprimée}

Date de publication : 1 décembre 2007

Pagination : 33-37

ISSN : 0395-773X

\section{Référence électronique}

Céline Château, « La traduction de qualité : un point de vue du Bureau Permanent de la Conférence de La Haye de droit international privé », Traduire [En ligne], 215 | 2007, mis en ligne le 01 décembre 2007, consulté le 19 avril 2019. URL : http://journals.openedition.org/traduire/1295 ; DOI : 10.4000/ traduire. 1295 


\section{La traduction de qualité : un point de vue du Bureau Permanent de la Conférence de La Haye de droit international privé}

La Conférence de La Haye de droit international privé est une organisation intergouvernementale de presque 70 membres, dont la Communauté européenne, qui a commencé ses travaux en 1893, lorsque le français était le vecteur privilégié de la diplomatie. Devenue bilingue anglais-français au début des années 60, la Conférence évolue désormais dans un monde où l'anglais fait figure de novlangue orwellienne, dans les relations internationales tout au moins. Rien de bien original à première vue, mais ce rappel historique est important pour situer les besoins de traduction du secrétariat de la Conférence, le Bureau Permanent, au-delà de la spécificité de la matière même, le droit international privé.

\section{L'esprit du bilinguisme}

Les textes des Conventions de La Haye produits au sein de la Conférence sont depuis quelques décennies authentiques dans ses deux langues officielles, l'anglais et le français. Les négociations se déroulent en deux langues et la rédaction proprement dite s'élabore en comités bilingues. Mais la méthode se heurte de plus en plus au principe de réalité, à savoir que les délégués des États membres parlent de moins en moins le français, alors que l'anglais donne à un grand nombre le sentiment de se comprendre. La tentation de ne parler qu'anglais en séance, pour produire un texte qui n'aura plus qu'à être traduit en français, est grande, a fortiori dans des comités de taille restreinte. Pourquoi s'embarrasser de discussions en deux langues, impliquant des explications souvent lourdes entre représentants de différentes communautés linguistiques, dont une partie est souvent exclue faute de connaître l'autre langue, alors que le résultat sera le même?

En fait, le processus de rédaction dans une langue, avec traduction dans une autre, est plus rapide a priori qu'une rédaction bilingue, mais 
l'efficacité est loin d'être garantie. La rédaction bilingue des conventions permet à une langue de nourrir l'autre, le questionnement dans l'une faisant souvent surgir les imprécisions de l'autre. La Conférence de La Haye tient donc à maintenir sa méthode de rédaction simultanée dans ses deux langues officielles, même si dans les faits, il lui faut parfois recourir à une traduction a posteriori, certes effectuée en interne, par des représentants des États membres et des fonctionnaires du Bureau Permanent. L'important reste que le texte original peut être modifié, si nécessaire, au vu du travail de traduction qui fait bien partie intégrante du processus de rédaction.

\section{Le traducteur-rédacteur}

Ce processus de rédaction bilingue se limite aux textes des conventions elles-mêmes et à quelques conclusions adoptées en fin de réunion, mais son esprit imprègne l'ensemble des documents publiés par le Bureau Permanent, pour lesquels il a recours à des traducteurs indépendants. S'il s'agit bien de traduction juridique, on est pourtant loin des travaux que peuvent avoir à effectuer des traducteurs assermentés, comme des traductions de jugement ou de pièces d'état civil, dont le contenu original ne sera en rien influencé par la traduction. La démarche proposée par le Bureau Permanent est plus dynamique et demande plus d'effort de part et d'autre de la relation client-traducteur.

Pour certains clients, il est essentiel de limiter les questions ouvertes afin de recevoir un texte qui sera éventuellement utilisable directement après réception de la traduction. Les traducteurs du Bureau Permanent, internes comme externes, sont au contraire vivement encouragés à souligner les imprécisions, erreurs et éventuelles incohérences des rapports et documents qui leur sont soumis. Si le traducteur se contente de traiter le document présenté sans esprit critique, en collant au texte et en essayant d'interpréter des idées peu claires à l'origine, alors son travail ne sera pas considéré comme une bonne traduction.

Travaillant sur des textes juridiques, outre leur spécialisation terminologique et leur aptitude à consulter différentes sources pertinentes, les (bons) traducteurs de la Conférence de La Haye de droit international 
privé sont ainsi des interlocuteurs directs des juristes chargés des projets de rédaction des conventions ou des rapports qui les accompagnent. Leurs interrogations amènent régulièrement les auteurs à réviser leur texte, sur la forme comme sur le fond, à la marge certes mais sur des points parfois très importants.

\section{Concision, précision, rapidité}

Le dialogue avec l'auteur n'exonère pas toutefois le traducteur de son travail de recherche terminologique. Le droit, qui plus est le droit international privé, est un domaine où concision et précision sont essentielles. Pour travailler pour le Bureau Permanent, les traducteurs en arrivent souvent à compléter leur formation en droit ou à améliorer leur connaissance du registre. La tâche est compliquée ici par la diversité des contextes dans lesquels des termes identiques sont utilisés, pour recouvrir des réalités différentes. Certes, par tradition, la Conférence de La Haye tend à suivre les règles du "français de France ", tout comme, pour ses travaux en anglais, elle se réfere à l' " anglais britannique ". Toutefois, il lui faut permettre à toutes les personnes parlant une (presque) même langue de comprendre les concepts évoqués dans ses documents, a fortiori dans les conventions qui auront un jour force de loi dans différents pays. Alors, au-delà des langues, il faut faire comprendre des réalités juridiques différentes. Le " notary " américain exerce une autre profession que le notaire français, mais il est très tentant de traduire son titre littéralement. Autres exemples classiques, le titre d' "Attorney general " qui, d'un pays anglophone à l'autre, désigne soit un procureur, soit un haut conseiller juridique ou encore le ministre de la Justice, ou bien la notion de "legal aid ", aide juridique, qui selon les pays peut dépasser ou se limiter à l'aide juridictionnelle. Les notes peuvent ici s'avérer très utiles, par exemple pour expliciter le contenu d'un terme paraissant évident vu d'un certain pays mais qui recouvre une réalité différente dans un autre pays où l'on parle pourtant la même langue.

Autre aspect important : le temps. La tentation est souvent grande, en effet, de diffuser les textes et les documents produits au Bureau 
Permanent dans la langue de rédaction originelle (l'anglais, dans l'immense majorité des cas), souvent sous forme provisoire mais avant même que la traduction ne soit effectuée. Cette dérive a bien sûr des conséquences en matière de plurilinguisme et de multiculturalisme (les francophones destinataires des textes auront souvent intérêt, pour pouvoir consulter et débattre rapidement avec leurs homologues anglophones, à ne pas attendre la traduction et lire le texte en anglais), mais d'un point de vue strictement pragmatique, cette course contre la montre limite en fait le rôle de la traduction dans le processus de rédaction. Difficile en effet de changer le texte initial déjà diffusé, même si le travail de traduction a fait émerger des inexactitudes.

\section{Garder le cap}

Traduire pour la Conférence de La Haye signifie donc souvent traduire en urgence, en tout cas rapidement, tout en faisant preuve de précision, de concision, et en n'hésitant pas à exercer son esprit critique dans un dialogue avec les auteurs. Un tel niveau d'exigence conduit logiquement à instaurer des relations à long terme avec les traducteurs. Le flux de documents à traduire par le Bureau Permanent est trop irrégulier pour justifier l'emploi exclusif de traducteurs internes, d'où le recours à des indépendants. La continuité de la relation est toutefois essentielle, pour assurer et rentabiliser l'acquisition de connaissances terminologiques, mais aussi pour établir des relations de confiance qui vont au-delà de la production isolée d'une traduction.

Le Bureau Permanent a la chance d'avoir trouvé des interlocuteurs de qualité, mais l'expansion de l'Organisation et l'irrégularité de la production des documents conduit inéluctablement à une quête quasi perpétuelle de nouveaux collaborateurs, pour lesquels le flux de travail ne sera pas forcément suffisant pour assurer une relation durable. Sans compter qu'une troisième langue de travail, l'espagnol, commence à s'imposer de plus en plus aux côtés des deux langues officielles, pour permettre la participation bienvenue des pays d'Amérique, qui bien souvent ne maîtrisent ni l'anglais ni le français. Les principes exigeants qui guident les travaux en français et anglais devraient trouver natu- 
rellement leur prolongement en espagnol. Mais le dialogue nécessaire entre les traducteurs et les membres du Bureau Permanent ne se fait pas aussi facilement avec une troisième langue, non officielle et dont la maîtrise n'est donc pas une condition du recrutement au sein de l'Organisation. L'expansion de l'Organisation, notamment géographique et linguistique, implique donc des ajustements qui dépassent le seul recours à un nombre accru de traducteurs externes. La problématique de la traduction de qualité, au-delà du bilinguisme, n'a pas fini d'occuper les fonctionnaires du Bureau Permanent et les traducteurs externes sur lesquels ils s'appuient.

Les opinions exprimées dans cet article n'engagent que leur auteur et ne prétendent pas refléter celles du Bureau Permanent de la Conférence de La Haye de droit international privé.

Céline Château

Responsable de l'administration Bureau Permanent de la Conférence de La Haye de droit international privé

\section{De l'intérêt de la langue maternelle}

La règle selon laquelle il faut toujours traduire vers sa langue maternelle a un fondement très logique dans la mesure où les psychologues font une importante distinction entre deux formes de mémoire : la mémoire de rappel et la mémoire de reconnaissance.

La mémoire de reconnaissance correspond au vocabulaire passif (on reconnaît les mots quand on les voit). La mémoire de rappel correspond au vocabulaire actif : on se rappelle les mots et on les utilise sans avoir besoin d'une aide quelconque. La capacité de reconnaissance d'un individu est bien plus importante que sa capacité de rappel, qu'il s'agisse de sa langue maternelle ou d'une langue étrangère apprise. De ce fait, il n'est pas très pertinent de dire qu'un individu moyen dispose d'un "vocabulaire " de $x$ milliers de mots sans préciser si l'on parle de sa mémoire de rappel ou de reconnaissance. 